\title{
The ISO-SWS post-helium atlas of near-infrared stellar $\operatorname{spectra}^{\star, \star \star, \star \star \star}$
}

\author{
B. Vandenbussche ${ }^{1}$, D. Beintema ${ }^{3}$, T. de Graauw ${ }^{3}$, L. Decin ${ }^{1, \dagger}$, H. Feuchtgruber ${ }^{4}$, A. Heras ${ }^{5}$, D. Kester ${ }^{3}$, F. Lahuis ${ }^{3}$, \\ A. Lenorzer ${ }^{2}$, R. Lorente ${ }^{6}$, A. Salama ${ }^{6}$, C. Waelkens ${ }^{1}$, L. Waters ${ }^{1,2}$, and E. Wieprecht ${ }^{4}$ \\ 1 Instituut voor Sterrenkunde, K.U.Leuven, Celestijnenlaan 200B, 3001 Leuven, Belgium \\ 2 Sterrenkundig Instituut "Anton Pannekoek", Amsterdam, The Netherlands \\ 3 SRON National Institute for Space Research, Groningen, The Netherlands \\ ${ }^{4}$ Max-Planck-Institut für Extraterrestrische Physik, Garching, Germany \\ 5 Astrophysics Missions Division, Research and Scientific Support Department of ESA, ESTEC, PO Box 299, \\ 2200 AG Noordwijk, The Netherlands \\ 6 ISO Data Center, Astrophysics Division of ESA, Villafranca del Castillo, Spain
}

\section{Received 11 March 2002 / Accepted 15 May 2002}

\begin{abstract}
We present an atlas of near-infrared spectra $(2.36 \mu \mathrm{m}-4.1 \mu \mathrm{m})$ of $\sim 300$ stars at moderate resolution $(\lambda / \delta \lambda \approx 1500-$ 2000). The spectra were recorded using the Short-Wavelength Spectrometer aboard the Infrared Space Observatory (ISO-SWS). The bulk of the observations were performed during a dedicated observation campaign after the liquid helium depletion of the ISO satellite, the so-called post-helium programme. This programme was aimed at extending the MK-classification to the nearinfrared. Therefore the programme covers a large range of spectral types and luminosity classes. The $2.36 \mu \mathrm{m}-4.05 \mu \mathrm{m}$ region is a valuable spectral probe for both hot and cool stars. H I lines (Bracket, Pfund and Humphreys series), He I and He II lines, atomic lines and molecular lines $\left(\mathrm{CO}, \mathrm{H}_{2} \mathrm{O}, \mathrm{NH}, \mathrm{OH}, \mathrm{SiO}, \mathrm{HCN}, \mathrm{C}_{2} \mathrm{H}_{2}, \ldots\right)$ are sensitive to temperature, gravity and/or the nature of the outer layers of the stellar atmosphere (outflows, hot circumstellar discs, etc.). Another objective of the programme was to construct a homogeneous dataset of near-infrared stellar spectra that can be used for population synthesis studies of galaxies. At near-infrared wavelengths these objects emit the integrated light of all stars in the system. In this paper we present the dataset of post-helium spectra completed with observations obtained during the nominal operations of the ISO-SWS. We discuss the calibration of the SWS data obtained after the liquid helium boil-off and the data reduction. We also give a first qualitative overview of how the spectral features in this wavelength range change with spectral type. The dataset is scrutinised in two papers on the quantitative classification of near-infrared spectra of early-type stars (Lenorzer et al. 2002) and late-type stars (Vandenbussche et al., in prep).
\end{abstract}

Key words. stars: general - infrared: stars - atlases

\section{Introduction}

Since the publication of the optical spectral atlas by Morgan et al. (1943), several authors have published spectral atlases of stars covering a broad range in spectral types and luminosity

Send offprint requests to: $\mathrm{B}$. Vandenbussche, e-mail: Bart.Vandenbussche@ster.kuleuven.ac.be

* Based on observations with ISO, an ESA project with instruments funded by ESA Members States (especially the PI countries France, Germany, the Netherlands and the United Kingdom) and with the participation of ISAS and NASA.

$\star \star$ The full atlas is available in electronic form at www. edpsciences.org

$\star \star \star$ Table 1 is only available in electronic form at the CDS via anonymous ftp to cdsarc.u-strasbg.fr $(130.79 .128 .5)$ or via http://cdsweb.u-strasbg.fr/cgi-bin/qcat?/A+A/390/1033

$\dagger$ Postdoctoral Fellow of the Fund for Scientific Research, Flanders. classes. With the development of spectrometers more sensitive towards the near-infrared and infrared, spectral atlases in these wavelength regions have become available. The IRAS LRS atlas (Olnon et al. 1986) contains more than 5000 low-resolution $(\lambda / \delta \lambda \approx 20-60)$ spectra between 7.7 and $22.6 \mu \mathrm{m}$. Recent work includes atlases of Fourier Transform Spectrograph spectra of MK-standards in the $H, J$, and $K$-band by Meyer et al. (1998); Wallace et al. (2000) and Wallace \& Hinkle (1996). Förster Schreiber (2000) published a library of near-infrared $K$-band spectra of 31 late-type giants and supergiants and two carbon stars at a spectral resolution of 830 and 2000 . The spectral library by Lançon \& Wood (2000) includes about 200 spectra between 0.5 and $2.5 \mu \mathrm{m}$ of cool, mostly variable, giants and supergiants. Heras et al. (in prep) describe the mid-infrared spectral characterisation of stars without dust excess using full ISOSWS spectra at low resolution $(\lambda / \delta \lambda \approx 300)$, most of which were obtained in the "STARTYPE" ISO observing programme. 
An extension of the "STARTYPE" project with the classification of all SWS full spectra contained in the ISO archive is in preparation.

A moderate resolution spectral atlas of stars between 2.3 and $4 \mu \mathrm{m}$, covering a broad range in spectral type and luminosity classes is not available. This wavelength range is accessible from the ground, but it is seriously contaminated by strong telluric lines, e.g. of $\mathrm{H}_{2} \mathrm{O}$.

This was the prime scientific driver of the ISO-SWS posthelium programme. The Infrared Space Observatory (ISO) was launched on November 17th, 1995. In April 1998, the end of the ISO operations was announced by the depletion of the liquid helium used for the cooling of the telescope and the focal plane instruments. After the liquid helium depletion of ISO, an engineering test programme was conducted on the different satellite systems. During this test programme the temperature of the focal plane remained sufficiently low to operate the InSb band 1 detectors of the SWS. These detectors are photodiodes, whereas the other SWS detectors are photoconductors which need low temperatures to limit the dark currents.

The instrument could be operated in some time slots available during the engineering test programme. This observing time was granted to a programme aimed at extending the MKclassification to the near-infrared. Despite various visibility constraints and other technical complications related to the operation of the instrument beyond nominal circumstances, the recorded spectra of 238 stars yield a signal-to-noise better than 10 across the entire spectrum. This has been our selection criterion to include the spectra in the dataset. Low scheduling priority was given to the programme stars that were already observed at a similar resolution during the nominal part of the mission in the framework of various other programmes. The dataset has been completed with these 55 nominal-phase spectra.

The post-He programme is complementary to the ISO STARTYPE programme. The goal of the STARTYPE proposal was to construct a data-base for spectral classification of the brightest representative sources in the IRAS Low Resolution Spectrometer (LRS) classification, the NASA Ames AI classification of the LRS and additional sources based on MK spectral type or IRAS colour. The core STARTYPE observation programme consisted of about 100 low resolution SWS AOT1 spectra $(2.36-45 \mu \mathrm{m})$ and PHT-S spectra $(2.47-4.87 \mu \mathrm{m}, 5.84-$ $11.62 \mu \mathrm{m})$ of sources of different spectral type, both with and without dust excess. This sample is currently being extended with all SWS AOT1 observations of stars observed during the entire ISO lifetime (Sloan et al., in prep.) The post-He programme covers a bigger sample (293 spectra) at higher spectral resolution in the wavelength range $2.36-4.05 \mu \mathrm{m}$. The wavelength range does not allow to characterise the dust excess of the stars across different MK spectral types and LRS classes but the higher spectral resolution allows to characterise more accurately the strength of diagnostic spectral features in this wavelength range.

In this paper we present the spectral atlas of 293 stars.

In Sect. 3 we describe the ISO-SWS instrument settings and observation modes used. In Sect. 2 we outline the source selection criteria and list the sources observed. In Sect. 4 we describe the calibration of the ISO-SWS instrument after the liquid helium depletion. The data reduction steps are discussed in Sect. 5. In Sect. 6 we travel through the HR-diagram and show qualitatively how the strength of spectral features varies in different spectral types and luminosity classes. Section 7 describes how the atlas and the reduced data can be retrieved electronically from the ISO Data Centre.

\section{Source selection}

The sources in the post-helium observation program were selected on the basis of three criteria:

1. The ISO visibility around the expected helium boil-off date. The predictions of this date had an estimated accuracy of \pm two weeks.

2. The brightness of the source. Since the integration time of the post-He observation template is fixed, the signal-tonoise ratio is determined by the brightness of the source. Therefore only sufficiently bright stars are in the sample ( $>1$ Jy across the entire wavelength range covered).

3. Covering a large range of spectral types and luminosity classes.

The programme stars were selected primarily from The Bright Star Catalogue (Hoffleit \& Warren 1991) according to these criteria. The Wolf-Rayet stars were selected from van der Hucht et al. (1997). Many of the selected stars had been observed already during the nominal operations phase of the ISO satellite. We avoided the duplication of these observations during the post-helium phase. Instead, the spectra of 55 programme stars obtained during the nominal phase were added to the final dataset.

The complete dataset contains 293 measurements. Those are the selected measurements of which the signal to noise ratio is better than 10, allowing the identification of the molecular and atomic spectral features. Figure 1 shows a HR-diagram for the programme stars. The sample covers spectral types between O4 and M9 and luminosity classes from the main-sequence to supergiants.

Table 1 (available electronically at CDS) lists the sources in our sample together with their spectral type, ISO observation identification (TDT), observation mode and the integration time.

\section{Observations}

The spectra in the atlas were measured with the ShortWavelength Spectrometer SWS (de Graauw et al. 1996) aboard the Infrared Space Observatory ISO (Kessler et al. 1996). The bulk of the observations in the atlas were performed after the helium boil-off of the satellite. These observations were executed using a dedicated engineering observation mode, the socalled post-helium observation template.

The astronomical observation templates (AOT) of the nominal operations phase were not appropriate for the post-helium observations. A special observation mode was designed to avoid unnecessary energy dissipation by the long wavelength grating scanner and the heaters in the read-out electronics of 


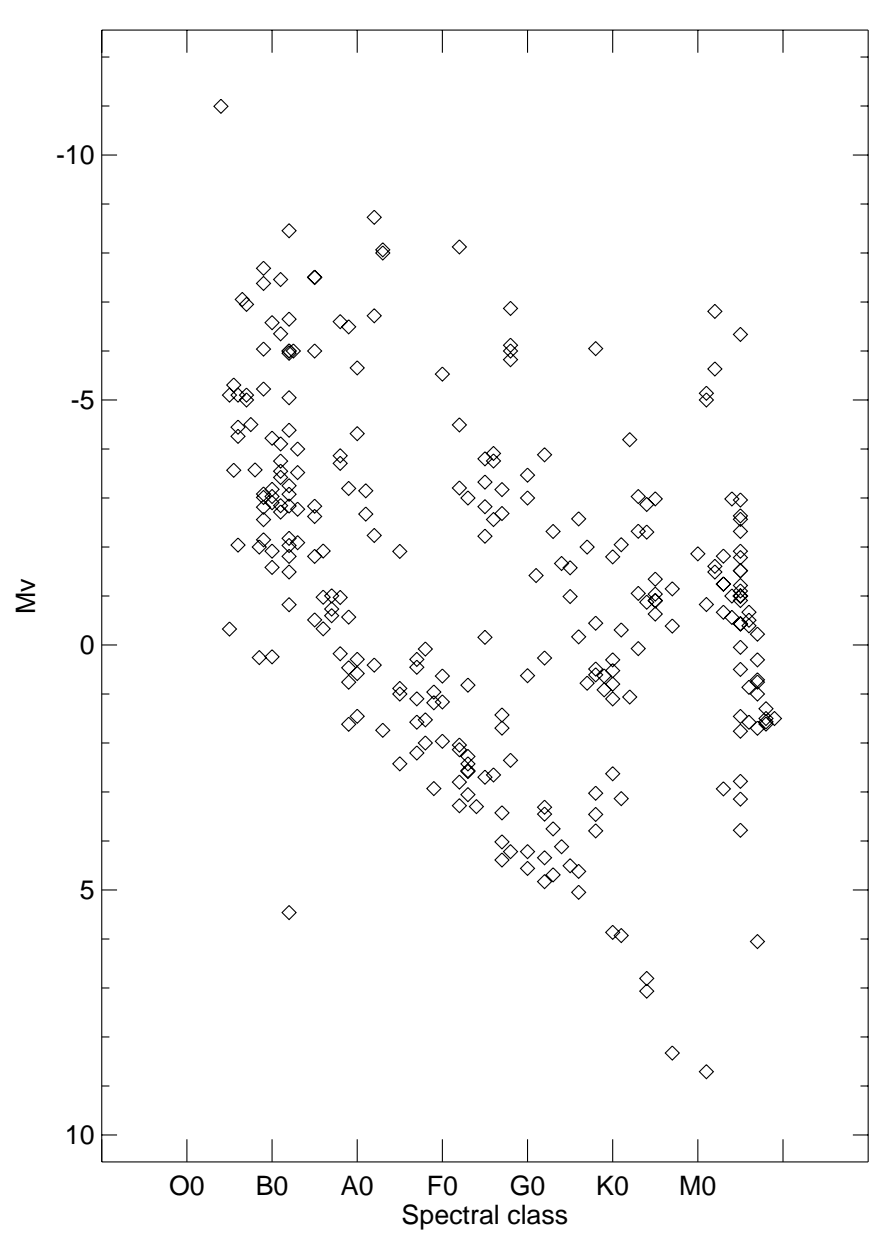

Fig. 1. Hertzsprung-Russell diagram of the programme stars. Spectral classes are taken from the literature; absolute $V$ magnitudes are calculated from Hipparcos parallaxes and photometry. No bolometric correction was applied. For WR-stars, carbon stars and other stars without reliable photometry or distance the approximate location is indicated.

the detector blocks not used. The grating scanning ranges were limited to the angles corresponding to the wavelengths covered in AOT-bands $1 \mathrm{~A}$ to 1E. The observation was implemented and tested in the Calibration Uplink System (CUS). Therefore posthelium observations appear in the ISO archive with observation type "S99".

The observation mode existed in two flavours. The fast version had a continuous scanning strategy like the nominal AOT1 speed 4 observation mode with a dwell time of $1 / 4 \mathrm{~s}$. The ontarget-time was $2352 \mathrm{~s}$. The slow version was scanning like in an AOT 1-like observation but with a dwell time of 1/2 s. It lasted for $4408 \mathrm{~s}$. The slow version was used for fainter sources.

The gains of the preamplifiers were set according to the expected flux of the observed source (gain 0 for sources brighter than $800 \mathrm{Jy}$, gain 1 for sources between $200 \mathrm{Jy}$ and $800 \mathrm{Jy}$, and gain 2 for sources fainter than $200 \mathrm{Jy}$ ). At the end of the observation a dark current measurement and an internal photometric calibration was done. The internal calibration source was used at its maximum brightness.

The complete time-line of an SWS post-helium observation is summarised below. In this time-line the wavelength scanning direction is expressed in grating scanner position readout units.
A scan up in grating position is a scan from long to short wavelengths and a scan down runs from short to long wavelengths.

1. Target Acquisition - point SWS aperture 1 to target.

2. Setup the amplifier gain for band 1 .

3. Single scan up band $1 \mathrm{~A}$.

4. Single scan down band $1 \mathrm{~A}$.

5. Single scan up band $1 \mathrm{~B}$.

6. Single scan down band $1 \mathrm{~B}$.

7. Point SWS aperture 2 to target.

8. Single scan up band 1D.

9. Single scan down band 1D.

10. Single scan up band $1 \mathrm{E}$.

11. Single scan down band $1 E$.

12. Dark current measurement.

13. Internal photometric calibration.

Table 2 lists the AOT-bands with the wavelengths covered, the integration times for the two speeds and the average spectral resolution in the band.

The sample contains 55 observations of stellar objects observed at a comparable resolution and wavelength coverage during the nominal operations of the ISO satellite. The observation modes meeting those criteria are the SWS AOT1 speed 4 observation mode and some AOT6 observations (de Graauw et al. 1996).

\section{The calibration of the SWS post-helium data}

During the test programme following the liquid helium depletion of ISO, the temperature of the focal plane remained sufficiently low to operate the InSb band 1 detectors of the SWS. As the temperatures of the focal plane increased, changes in the measured positions of the grating scanner mirror and changes in the response of the instrument could be seen. In this section we present the calibration of the changing characteristics of the instrument.

\subsection{Temperature change of the ISO focal plane}

The temperatures of the focal plane steadily increased after the helium boil-off. Figure 2 shows how after some days they exceeded the dynamic range of the temperature sensors on the spectrometer. The temperature trend of the focal plane later in the post-helium phase can be inferred from a similar trend seen in the temperatures of the upper baffle of the ISO telescope. We estimate that the temperature of the InSb detectors increased from the nominal operating temperature about $3.5 \mathrm{~K}$ to $40 \mathrm{~K} \pm 5 \mathrm{~K}$ over the 30 days of post-He operations.

Before the helium boil-off, it was anticipated that the spectrometer might reach a temperature where the JFET cryogenic read-out would function better with the heaters switched off (the heaters raise the JFET temperature by about $70 \mathrm{~K}$ whilst the focal-plane temperature increased by $45 \mathrm{~K}$ ). The slow temperature increase however made heating required throughout the entire post-helium phase for the JFETs to function properly. 
Table 2. Overview of the four spectral bands, their wavelength coverage, average spectral resolution and the integration times for the fast, resp. slow version of the SWS post-helium astronomical observation template.

\begin{tabular}{ccccc}
\hline \hline AOT-band & Wavelength range & Average Resolution $(\lambda / \delta \lambda)$ & $T$ Int fast & $T$ Int slow \\
\hline 1A & $2.37-2.61 \mu \mathrm{m}$ & 1800 & $390 \mathrm{~s}$ & $782 \mathrm{~s}$ \\
1B & $2.59-3.04 \mu \mathrm{m}$ & 1500 & $534 \mathrm{~s}$ & $1070 \mathrm{~s}$ \\
1D & $3.01-3.53 \mu \mathrm{m}$ & 1800 & $652 \mathrm{~s}$ & $1306 \mathrm{~s}$ \\
1E & $3.51-4.09 \mu \mathrm{m}$ & 1300 & $472 \mathrm{~s}$ & $946 \mathrm{~s}$ \\
\hline
\end{tabular}

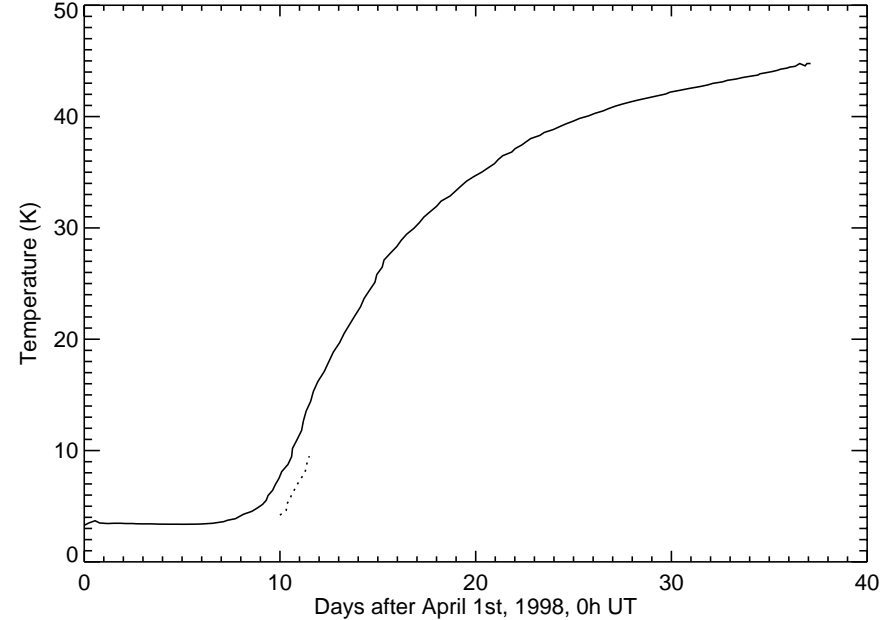

Fig. 2. The temperatures of the optical support structure (lower short curve, dashes) and of the upper baffle of the ISO telescope (upper curve, solid line) increased smoothly during the post-He phase.

This was verified during every revolution with SWS operations. The instrument controllers manually up-linked a command sequence to the spacecraft to switch off the JFET-heaters for one minute. It was verified offline that this indeed corrupted the detector read-out.

\subsection{Wavelength calibration and spectral resolution}

As the focal plane temperatures of ISO increased, a distinct shift in the position of spectral features in the SWS spectra was seen. The spectral shift is almost certainly caused by a small displacement of the coils of the electromagnetic position sensor on the scan mirror. Figure 3 shows how the position of the hydrogen Pfund Beta line shifts in the un-calibrated spectrum of Alpha Lyrae.

The wavelength calibration of the SWS was tied to measurements of absorption lines in vapour cells in the laboratory and strong emission lines in astronomical sources. To facilitate the wavelength calibration of the wavelength regions with little or no lines in these measurements, the fringe spectrum from the internal calibration source is measured. An internal wavelength calibrator in the spectrometer served as a wavelength reference between external calibrations. This wavelength calibrator consists of a continuum radiator behind a window that serves as a fixed Fabry-Perot etalon. During the post-helium operations, a spectrum of the fringes produced by this system was observed at the beginning of every revolution when the SWS was switched on.

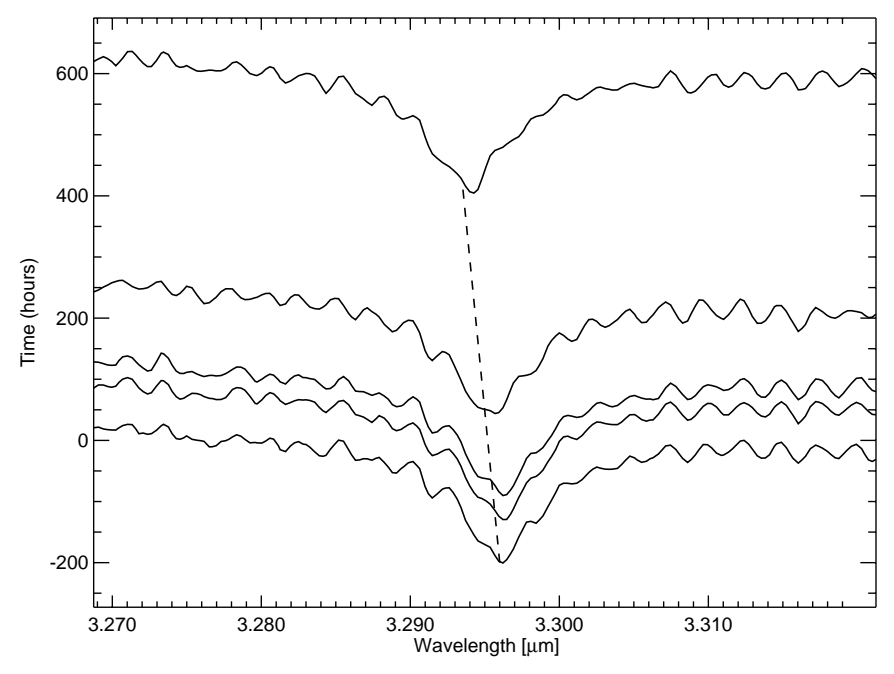

Fig. 3. As temperatures changed, geometry changes in the grating scanner mechanism induced a shift in the position of spectral features. We see the hydrogen Pfund Beta line in the spectrum of Alpha Lyrae. The time between the measurements was used to offset the different spectra in the figure.

Each of these measurements was used to determine a wavelength calibration of the SWS, applicable for the instrument at the time of the calibration measurement. The wavelength calibration for a single post-He observation can be calculated by interpolating these calibration parameters in time.

During the major part of the ISO mission, the planetary nebula NGC 6543 was visited every two weeks for an AOT2 scan of selected lines. These measurements were used as an external check of the wavelength calibration stability. To allow a similar monitoring of the wavelength calibration stability and to verify the spectral resolution during the post-helium phase, a Calibration Uplink System (CUS) observation mode was engineered to perform line scans similar to the AOT2 scanning mode.

The accuracy of the wavelength calibration during the nominal part of the ISO mission is better than 1 step in the position read-out of the grating scanning mirrors. In the shortwavelength section this translates to an accuracy of $\lambda / 5000$ $\lambda / 12000$ (Valentijn et al. 1996). Verification of the line positions in the NGC 6543 observations indicate that the accuracy of the post-helium wavelength calibration is better than $\lambda / 4000$.

Line widths determined on unresolved lines in the spectrum of NGC 6543 varied throughout the post-He mission, but within the scatter of similar measurements in the normal mission. The observed scatter is probably due to pointing jitter. Figure 4 depicts the variation of the resolution of the unresolved hydrogen Pfund $\delta$ line at $3.297 \mu \mathrm{m}$ in the planetary 


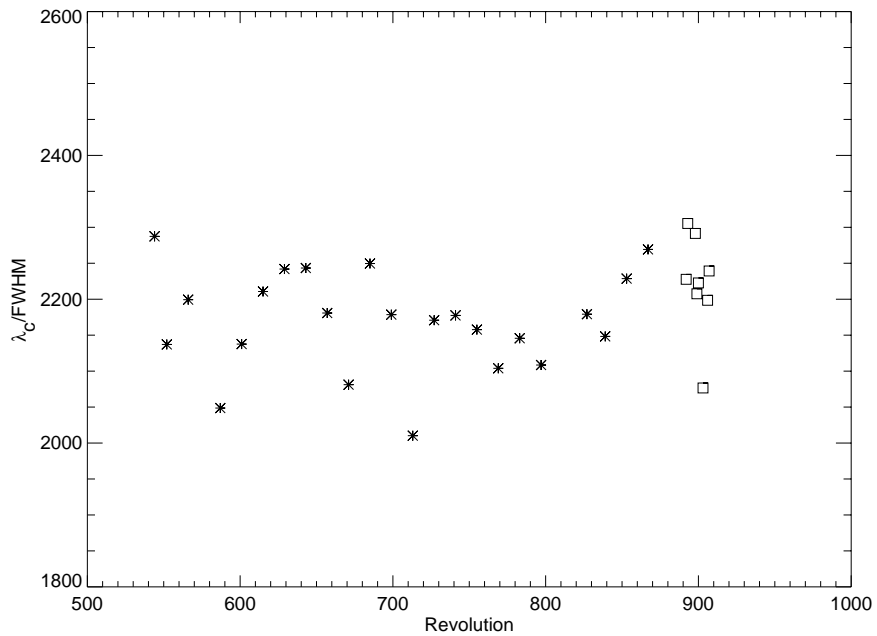

Fig. 4. Spectral resolution from the line-widths observed on the H Pfund $\delta$ line [3.297 $\mu \mathrm{m}]$ in NGC 6543. Asterisks are the values during the nominal mission. the squares are the values measured during the post-He phase. The lines were scanned using the AOT2 observation mode or an equivalent engineering mode after helium boil-off.

nebula NGC 6543. One can see that the resolution during the post-He phase is not drastically different from the values during the nominal mission (Valentijn et al. 1996). The spectral resolving power of the AOT2 observation mode and the equivalent post-helium engineering observation mode are the true instrumental resolution whereas the resolution of the AOT 1 observations and the post-helium observations is limited by the sampling (Leech et al. 2002).

\subsection{Detector dark currents}

The dark signals and the noise on the dark signals measured after the helium boil-off are not significantly different from the nominal mission. Figure 5 shows the mean of the detector signals measured during the dark current measurements in the calibration observations during every revolution in the post-He phase. There is no distinct trend with the temperature increase. This is not surprising, since the InSb photodiodes operate without bias voltage and so by their nature produce no dark current.

\subsection{Relative spectral response change}

In every post-He revolution with SWS observations, a full spectrum of at least one calibration star was measured. Section 4.6 gives an overview of the calibration sources used for the flux calibration (both relative and absolute) of the post-helium phase. We have chosen calibration sources for which reliable reference spectral energy distributions (SEDs) are available. We have used synthetic spectra based on model atmospheres computed using the SOS-MARCS code (Decin 2000; Decin et al. 2000c, b, a).

Comparison of the observed calibration source spectra to a reference spectral energy distribution (SED) shows an increasing tilt of the observed spectrum as the temperature increases. This tilt is different in every AOT-band. As can be seen in

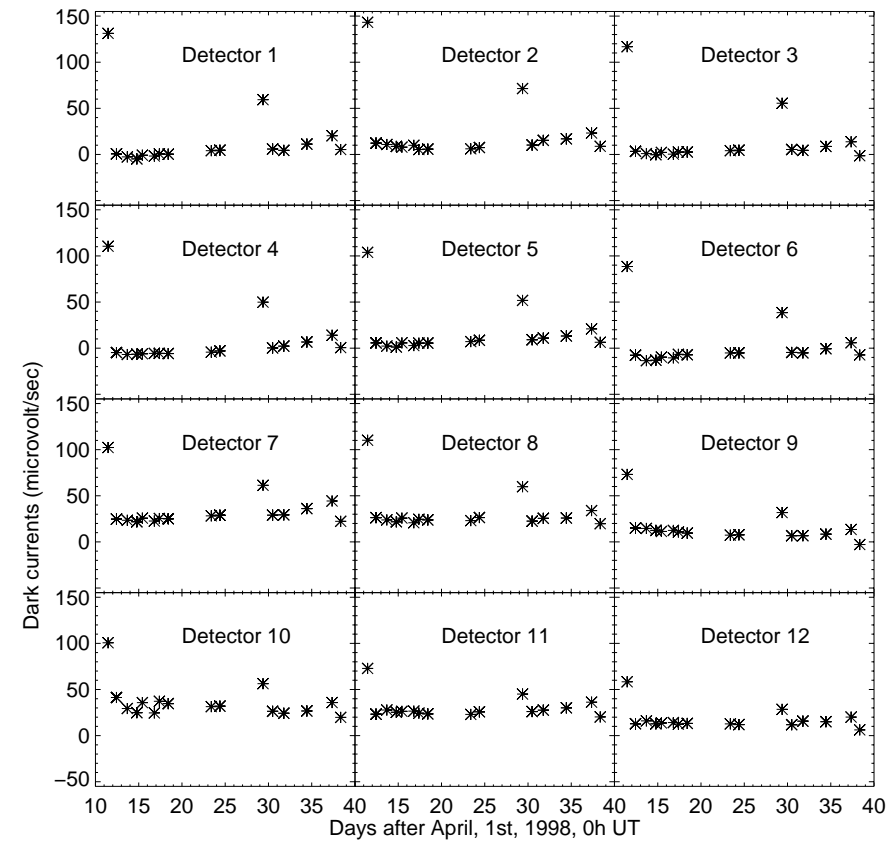

Fig. 5. The variation of the mean detector dark signals during the posthelium phase.

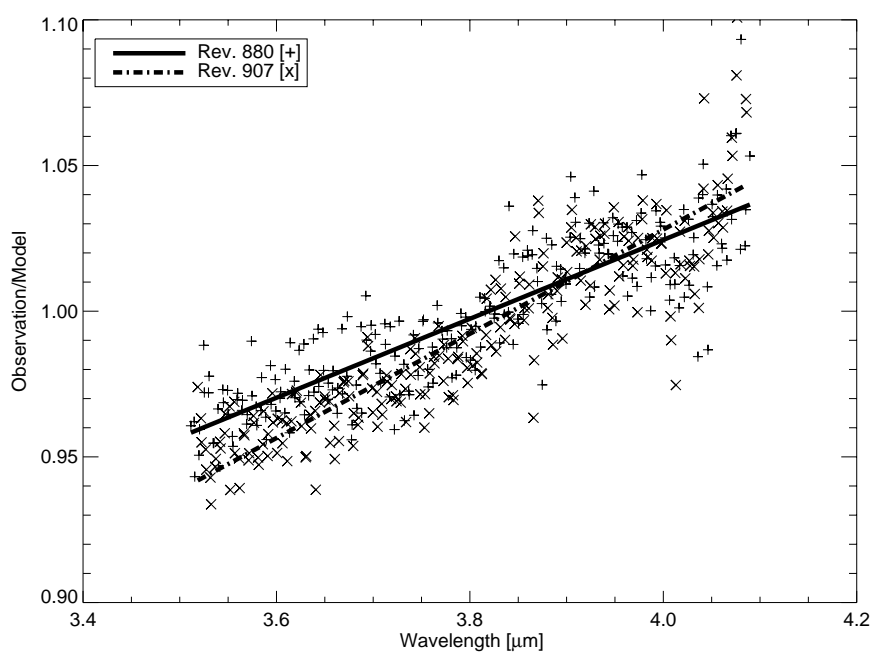

Fig. 6. Division of the observed spectrum of $\gamma$ Draconis by a synthetic spectrum in the beginning and the end of the post-helium phase. The Relative Spectral Response of the SWS tilts more as the temperature increases.

Fig. 6, if we divide the spectrum of $\gamma$ Draconis observed in revolution 880 and revolution 897 by the model SED, the linear fit to the residues shows a steeper slope in the latter revolution. The plot shows the changing slope of the Relative Spectral Response Function (RSRF) for band 1E. A similar effect is seen in the other 3 AOT-bands.

This effect was characterised for every spectral band. For every calibration observation we determined a linear tilt correction by fitting a straight line $R(\lambda)$ to the division of the measured spectrum by a model SED. The two coefficients of this function vary in time. We fitted a quadratic polynomial $P_{1}(t)$ and $P_{2}(t)$ to these coefficients as a function of time. The tilt to apply to a spectrum observed at time $t$ is then $P_{1}(t)+\lambda P_{2}(t)$. 

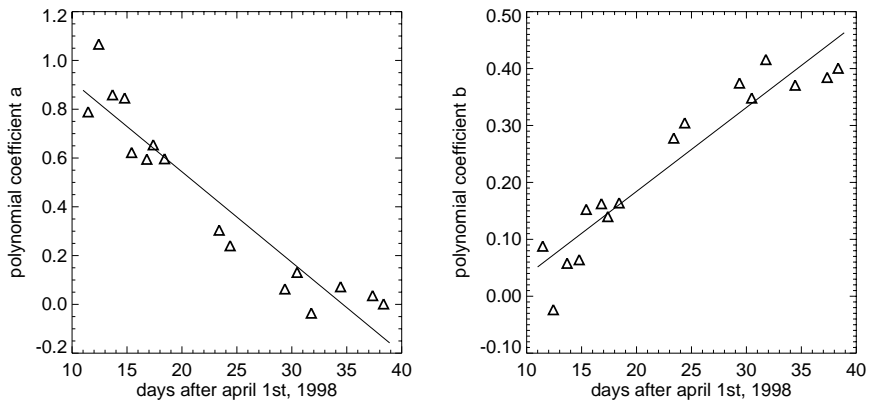

Fig. 7. Characterisation of the changing slope of the RSRF for detector 7 in AOT-band 1A. The triangles are the polynomial coefficients of the fits to the slope difference between calibration observations and model spectra. The solid line is a linear fit to the coefficients against time.

Figure 7 shows the coefficients of the fits to the RSRF residues and the fits to the change of these coefficients.

The characterisation described above minimises the risk of biasing the correcting tilt to a noisy observation or a specific spectral feature not accurately represented in the synthetic spectra. The different calibration stars cover a wide range in spectral types. The deficiencies in the synthetic spectra are of a different nature and are located in different spectral regions (see Sect. 4.6). The double fitting process described above results in some sort of "averaging out" of the uncertainties in the synthetic spectra and the observations.

In some post-helium spectra there is a hint of residual sinusoidal artefacts in the RSRF between 2.6 and 2.9 micron. The strength and the shape of these broad features varies between 0 and $2 \%$. The available calibration observations do not allow to improve the reliability of the RSRF in this area (see also Sect. 4.7 for a detailed analysis of the broadband accuracy of the post-helium spectra). This is the region where water bands have been observed in M stars (Ryde \& Eriksson 2002). Tsuji (2001) reports the detection of water in the $\mathrm{K}$ giant $\alpha$ Tau in the $6.2 \mu \mathrm{m}$ region of the ISO-SWS spectrum. The features we describe here are not related to these detections of $\mathrm{H}_{2} \mathrm{O}$ features in the nominal SWS spectra since the few post-helium spectra that show the features are of both late and early-type sources.

\subsection{Absolute flux calibration}

The SWS has built-in calibration sources to tie the flux calibration observations on external sources to frequent internal photometric calibration checks during every observation. During the post-helium phase the signal from these photometric checks increased with the increase of the focal plane temperature. However, the system response to the external calibration sources decreases with time (Fig. 8). This discrepancy can be understood as a combination of different effects. The increase of temperature of the calibration source could result in a higher flux. Also temperature changes of the telescope could result in a slight defocusing, which could explain (part of) the lower response to the external source. We believe the second effect is marginal, since no significant change in the wavelength resolution was seen (Sect. 4.2). Defocusing the telescope, i.e.

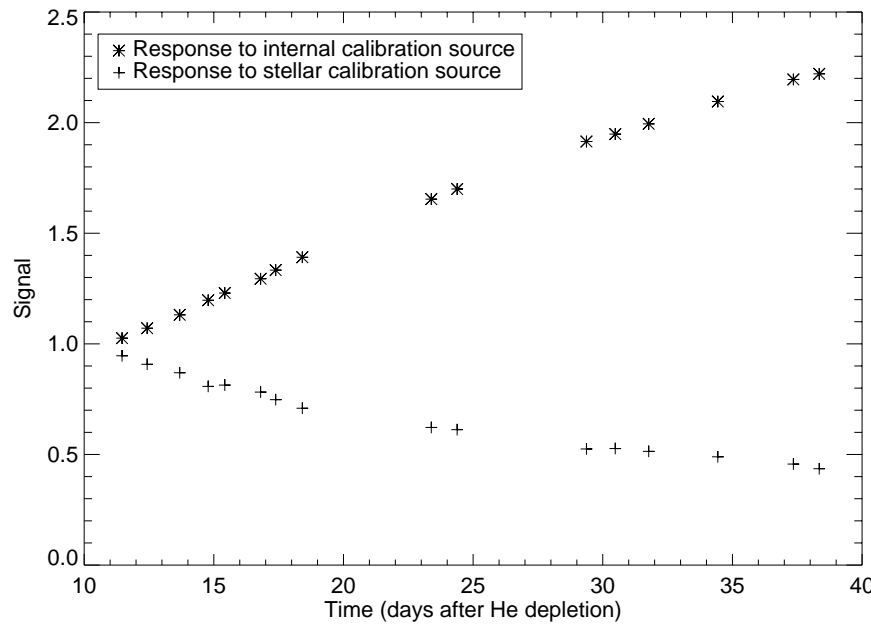

Fig. 8. Change of the SWS detector 7 response to the signal of the internal calibration source and to the signal of an external calibration star.

broadening the profile of the beam entering the instrument, would result in a broader instrumental profile.

The exact reasons for the system response changes cannot be disentangled, but the strategy for an empiric flux calibration is straightforward: for each spectral band signal/flux ratios per detector are determined from the daily observations of standard calibration stars. The calibration sources and model spectra used are listed in Sect. 4.6. A second order polynomial fit to those values against time is the flux calibration function. Evaluation of this polynome in the time of a post-helium observation gives the signal/flux ratio to apply to the observation.

Also here the method averages out uncertainties in the accuracy of spectral features in the individual model spectra and calibration observations.

\subsection{Post-helium flux calibration sources}

Table 3 shows the stars used to determine the tilt correction of the relative spectral response and the absolute flux calibration of the observations obtained during the post-helium phase. Choosing several calibration stars of different spectral type is of utmost importance. This avoids systematic flux calibration errors in spectral regions where the accuracy of the model spectrum of one source is less reliable.

The accuracy of the model spectra of late-type stars will typically be limited in the molecular absorption regions. The reasons for this are numerous: uncertain line opacities, mismatch between the actual instrumental profile and the profile used to convolve the high-resolution synthetic spectrum, etc. This is discussed in great detail by Decin (2000).

The weakest points in the model spectrum of $\alpha$ Lyrae will be of a different nature. The problems with e.g. Stark broadening that still persist in contemporary model atmosphere codes are not so important here because of the moderate resolution. Remaining problems can be expected in the strength of some atomic lines. The oscillator strengths of atomic lines in the infrared are not known to a high accuracy (Decin 2000). 
Table 3. The calibration stars used for the tilt correction of the relative spectral response and flux calibration of the post-helium phase.

\begin{tabular}{cccc}
\hline \hline Source & Sp. Type & \# obs & Reference SED \\
\hline$\alpha$ Lyr & A0 V & 6 & Decin (2000) \\
$\delta$ Dra & G9 III & 7 & Decin (2000) \\
$\gamma$ Dra & K5 III & 3 & Decin (2000) \\
\hline
\end{tabular}

Price et al. (2002) have recently questioned the use of synthetic spectra of late-type giants for the relative spectral response and absolute flux calibration of the SWS. They based their statement on the presence of artefacts in the SWS spectra of early-type stars between 4 and $12 \mu \mathrm{m}$ with the calibration of the ISO-SWS Off-line processing version 10.0. Two features of a few percent coincide with the location of the strong molecular absorption bands of $\mathrm{CO}[4.5 \mu \mathrm{m}]$ and $\mathrm{SiO}$ [8 $\mu \mathrm{m}]$ in the spectra of some of the calibration stars used. The calibration in the legacy version (10.1) of the SWS Offline Processing avoids the issue by only using calibration stars earlier than F9 between 4 and $5.5 \mu \mathrm{m}$ and between 7 and $10 \mu \mathrm{m}$. The conclusion of the authors that the use of synthetic spectra of cool giants needs further progress before they can be used for the calibration of ISO-SWS discounts the fact that the accuracy of other reference spectra (e.g. Cohen et al. 1999, and references therein) is not better than the synthetic spectra by Decin (2000). The use of late-type stellar calibrators for ISO-SWS is unavoidable since sensitivity and visibility constraints do not allow to calibrate the entire SWS wavelength range exclusively on early-type stars. The strengths of the molecular bands in the synthetic spectra over the wavelength range covered by the post-helium programme is also more reliable and have been verified by comparisons to high-resolution spectra obtained from the ground. Therefore the choice of calibration standards and the use of the synthetic spectra by Decin (2000) is appropriate for the flux calibration of the post-helium observations.

\subsection{Photometric accuracies}

In Table 4 we list the flux error budget of the post-helium observations and compare it to the error budget for the nominal phase derived from the RSRF accuracies and photometric error budget given in Leech et al. (2002). The question "What is the photometric accuracy of a spectrum" cannot be answered by a single number. The answer depends on the spectral scale. We have therefore disentangled the error on the integrated in-band flux of the entire spectrum, relative broadband errors within the spectra, the accuracy on a wavelength scale close to the spectral resolution and the error on the intensity of an unresolved line. The first error will be useful to estimate the accuracy of an in-band flux obtained with synthetic photometry on the posthelium spectra, the second error will be relevant when measuring the continuum shape or the strength of broad absorption features. The small-scale error gives an indication of the reliability of spectral features that are barely resolved and the latter error has to be accounted for when interpreting the measured intensity of an unresolved line.
A first contribution to the error on the total absolute average in-band flux in the spectrum is the accuracy of the flux calibration described in Sect. 4.5. It is determined by three factors: the uncertainties in the model SEDs, the noise in the individual flux calibration observations and the simplification of our responsivity change model (a second order polynomial). In Sect. 4.6 we have argued that the use of calibration sources of different spectral type minimises the contribution of the first factor. The magnitude of the two other contributions can be estimated from the difference between the response change as derived from the individual flux calibration observations and the response change model (Fig. 8). In the worst case this is $5 \%$. The other important contribution to the absolute integrated in-band flux error is the absolute pointing error of the satellite. The absolute pointing error of the ISO satellite after revolution 452 is $2^{\prime \prime}$. (Leech \& Pollock 2001). A rounding error in the observation definition of the first post-helium revolutions results in an additional offset between intended and actual pointing. In later post-helium revolutions technical tests on the attitude control system of the satellite to test algorithms with 2 or $1 \mathrm{gy}$ ros instead of 3 also make the pointing accuracy slightly worse. We therefore assume the absolute pointing error of our observations to be smaller than $2.5^{\prime \prime}$. The spatial response of the ISOSWS band 1 detectors has been characterised sufficiently well to know that in the worst case a pointing offset of $2.5^{\prime \prime}$ translates to a flux difference of $8 \%$ (Leech et al. 2002).

The broadband error is dominated by the accuracy of the RSRF and the simplification of the changing slope to model the change in the RSRF (Sect. 4.4). From the difference between the actual datapoints and the fit in Fig. 6 we estimate this error to be $5 \%$.

The third heading in our error bookkeeping is the smallscale error. In order to determine the dark noise in our observations we have replaced the signal in an observation by dark signals obtained during the instrument activation at the beginning of a revolution. Subsequently we have applied the same calibration and data reduction steps as applied on all the observations. This yields a spectrum where the noise around the zero-level is $1 \mathrm{Jy}$ in one resolution element as defined in Table 2. Another constraint on the small-scale accuracy is imposed by the RSRF. The ISO-SWS RSRF was determined prior to launch during the instrument tests in the laboratory. Full spectra of a cryogenic blackbody were measured at the full spectral resolution of the instrument. However, the blackbody source filled the aperture of the SWS completely, resulting in a slightly lower spectral resolution than obtained on point sources. Order selection filters in the optical train of band 1 produce small fringes that are marginally resolved in the laboratory RSRF. The broadband shape of the laboratory RSRF was further corrected based on in-orbit observations, but the accuracy of the RSRF on small spectral scale is still dominated by these unresolved fringes. It contributes another $1 \%$ to the small-scale error of the post-He spectra. In observations where the Absolute Pointing Error is large $\left(2.5^{\prime \prime}\right)$, the pointing jitter of about $0.5^{\prime \prime}$ around the absolute pointing direction results in a noise factor of about $2.5 \%$ (Leech \& Pollock 2001). With small Absolute Pointing error $\left(<1^{\prime \prime}\right)$, there is no significant effect from pointing jitter on the small-scale error. 
Table 4. The flux calibration error budget of the ISO-SWS posthelium observations and the nominal ISO-SWS observations.

\begin{tabular}{lcc}
\hline \hline Error source & Post-He & Nominal \\
\hline Absolute error on the average flux & & \\
Absolute flux conversion & $5 \%$ & $2 \%$ \\
Absolute Pointing error & $8 \%$ & $4 \%$ \\
\hline Relative errors - broadband & & \\
RSRF & $5 \%$ & $3 \%$ \\
\hline Relative errors - small scale & & \\
Dark noise & $1 \mathrm{Jy}$ & $1 \mathrm{Jy}$ \\
RSRF & $1 \%$ & $1 \%$ \\
Pointing jitter & $0-2.5 \%$ & - \\
\hline Line intensity of unresolved lines & & \\
Unresolved fringes & $20 \%$ & $20 \%$ \\
Linewidth jitter & $5 \%$ & $5 \%$ \\
\hline
\end{tabular}

We also list the error on the intensity of unresolved lines. We should expect instrumental fringes in the SWS band 1, even if we can not (or barely) see them in the spectra. Unresolved fringes would typically result in an intensity error of 10 to $20 \%$ of the measured intensity on an unresolved line. Figure 4 shows a jitter in the measured line-width of about $5 \%$, probably due to pointing errors in the dispersion direction of the spectrometer. This adds another 5\% uncertainty to the measured linewidth.

\section{Data reduction}

We have reduced the data within the SWS Interactive Analysis package (SIA) ${ }^{1}$.

The nominal SWS observations were processed with the algorithms and calibration parameters of the Off-Line-Processing pipeline OLP 10.

The changing characteristics of the SWS instrument during the post-helium phase required a different calibration. This is described in detail in Sect. 4.

All calibrated spectra have been reduced further in a consistent way. First a noise filter was applied. Data points that were more than 3 times the standard deviation away from the mean in a resolution bin were discarded. This process was repeated five times. The resolution bins were defined according to the average resolution per AOT-band as listed in Table 2.

Per AOT-band the data points were rebinned to the average resolution in the band (Table 2). In order to meet the Nyquist criterion for complete sampling of the measurement, we have oversampled four times when applying the noise filter and the rebinning.

Finally, small corrections to the absolute flux level of the four AOT-bands were applied to align them to each other. The data in the small overlap regions between the spectral bands was used to determine the scaling factors. The strategy we followed was to keep as many bands as possible fixed. The correction factors applied are small (mostly of the order of a few percent) and are listed in Table 1. The origin of the differences in the absolute flux level of the different bands lies in the fact that the contributions to the absolute error on the average flux

\footnotetext{
${ }^{1}$ IA3 is a joint development of the SWS consortium. Contributing institutes are SRON, MPE, KUL and the ESA Astrophysics Division.
}

as listed in Table 4 are different for different AOT-bands within one observation. Particularly the error introduced by pointing errors is inherently different for bands that use a different instrument aperture. Bands 1A and 1B use the same SWS aperture 1, while bands $1 \mathrm{D}$ and $1 \mathrm{E}$ employ aperture 2 . Switching to another aperture involves a change in the spacecraft attitude, so the pointing error can be different. The beam profiles of bands $1 \mathrm{~A}$ and $1 \mathrm{~B}$ are also different from the beam profiles of the other two bands, hence even the same pointing offset can result in a different scaling of the absolute flux level.

The data reduction applied was optimised to present a continuous spectrum at the highest defensible resolution. If accurate line intensities are needed, users of the post-helium data should revert to line-fitting procedures on unbinned spectra which we have made available in the ISO data Archive.

\section{Spectral features in the near-infrared spectra of stars}

The $2.36 \mu \mathrm{m}-4.05 \mu \mathrm{m}$ region is a valuable spectral probe for both hot and cool stars. HI lines (Bracket, Pfund and Humphreys series), $\mathrm{HeI}$ and HeII lines, atomic lines and molecular lines $\left(\mathrm{CO}, \mathrm{H}_{2} \mathrm{O}, \mathrm{NH}, \mathrm{OH}, \mathrm{SiO}, \mathrm{HCN}, \mathrm{C}_{2} \mathrm{H}_{2}, \ldots\right)$ are sensitive to temperature, gravity and/or the nature of the outer layers of the stellar atmosphere (outflows, etc.). In this section we give a qualitative discussion of the spectral features seen in the sources in our sample. We illustrate this with the spectra of representative sources in the atlas. For the spectral classes where several post-helium spectra are available we have selected the spectra with the best signal-to-noise.

Tables 5 and 6 show an overview of the total number of spectra in the atlas per spectral type.

\subsection{OB stars}

The spectra in the observed wavelength region of the normal OB-type giants and dwarfs show hydrogen lines in absorption of the Bracket $(\operatorname{Br} \alpha$ [4.0523 $\mu \mathrm{m}], \operatorname{Br} \beta$ [2.6259]), Pfund (Pf $\gamma[3.7406 \mu \mathrm{m}]-$ Pf22 [2.4036 $\mu \mathrm{m}]$ ) and Humphreys series (Hu14 [4.0209 $\mu \mathrm{m}]$ and higher). Some O supergiants show these hydrogen lines in emission and also show helium ionic lines (HeII(7-6) [3.0917 $\mu \mathrm{m}]$ ). The Be stars in the sample exhibit their hydrogen lines in emission. These lines originate from the gas in the circumstellar disk.

A detailed quantitative discussion of the spectra of OB-stars can be found in Lenorzer et al. (2002).

The spectra of Wolf-Rayet stars are characterised by various broad emission lines originating in the hot dense stellar winds that drive the extremely high mass loss of these stars. We see emission lines of HeI, HeII, CIII, CIV and the forbidden [CaIV] line at 3.21 $\mu \mathrm{m}$. (See also van der Hucht et al. 1996; Willis et al. 1997; Morris et al. 1999) A discussion of all ISOSWS observations of WR stars is in preparation by Morris et al.

Figure 9 shows the spectra of HD 190429 [O4If+], HD 30614 [O9.5] and HD 68273 [WC8] with the most prominent hydrogen and helium lines indicated. 


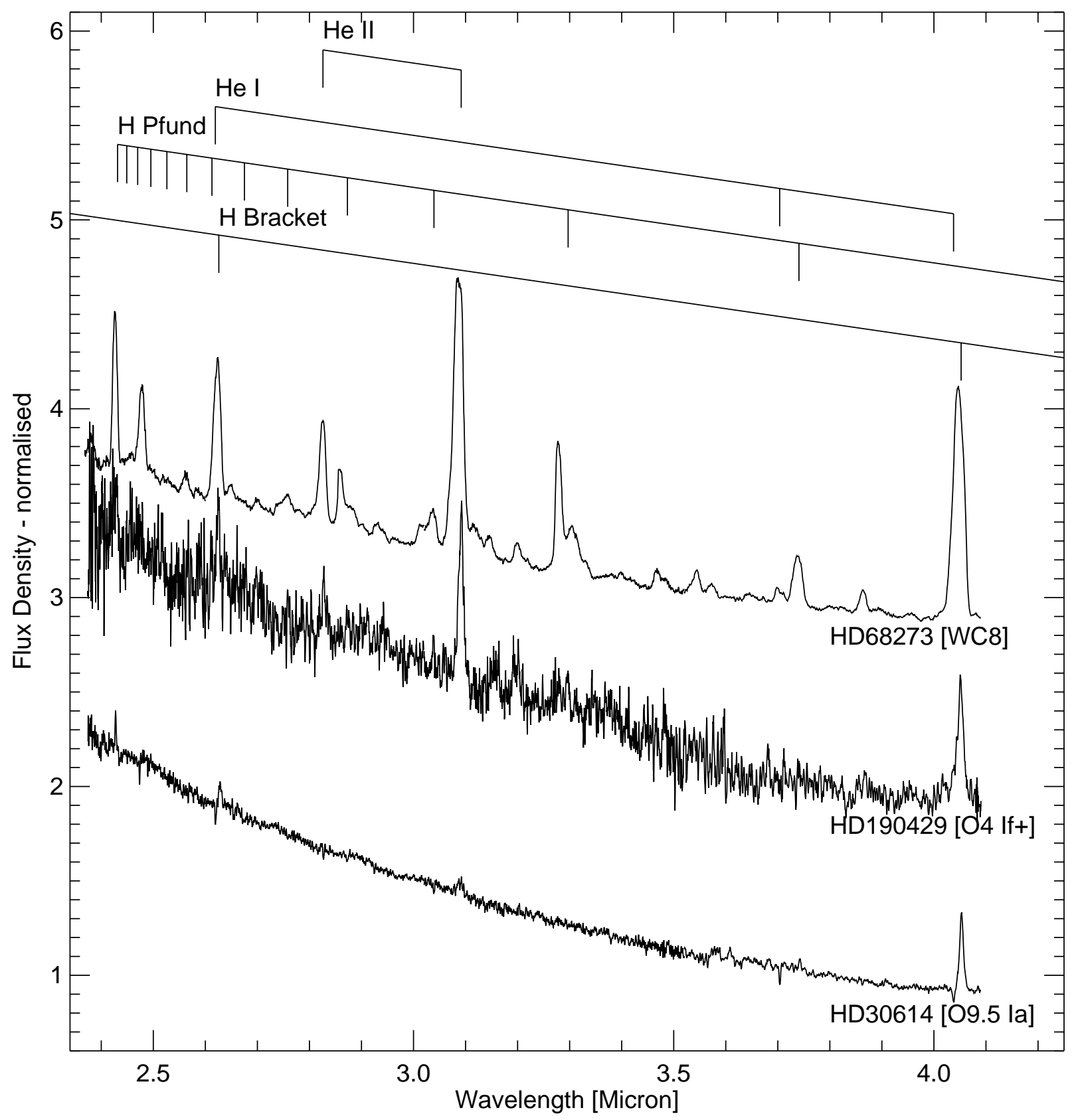

Fig. 9. Spectral features in the spectra of $\mathrm{O}$ and WR stars. The flux scale is in Jy, normalised to 1 at $3.8 \mu \mathrm{m}$ and offset for the different spectra.

In Fig. 10 we display the most prominent spectral lines in HD 93030 [B0Vp], HD 193924 [B2IV], HD 58350 [B5Ia], HD 196867 [B9IV] and HD 56139 [B2 IV-Ve].

\subsection{AF stars}

The near-infrared spectrum of A trough F-type stars is dominated by the HI lines of the Bracket $(n=4)$, Pfund $(n=5)$ and Humphreys $(n=6)$ series. We normally see those lines in absorption, except in some stars with a shell (e.g. Herbig Ae/Be stars) where the hydrogen emission originating in the shell can fill in some of the photospheric absorption (e.g. HD 190073).

Figure 11 shows a selection of spectra of A-F giants and supergiants while Fig. 12 displays a selection of A-F dwarfs.

\subsection{G stars}

In the spectra of G-type stars we see important contributions from various atomic lines. Towards later type G-stars the strength of the hydrogen absorption lines weakens and we start to see molecular species. The CO first overtone band appears between 2.36-2.45 $\mu \mathrm{m}$.

In Fig. 13 we present a selection of the spectra of $G$ dwarfs. Figure 14 shows a selection of the spectra of $\mathrm{G}$ supergiants.

\subsection{KM stars}

In cool stars $(\mathrm{K}, \mathrm{M})$ the spectrum shows atomic lines, but is dominated by molecular bands. We see the $\mathrm{CO}$ first overtone (2.36-2.45 $\mu \mathrm{m})$, the $\mathrm{OH}$ band $(3.02-3.4 \mu \mathrm{m})$, the $\mathrm{H}_{2} \mathrm{O}$ band 


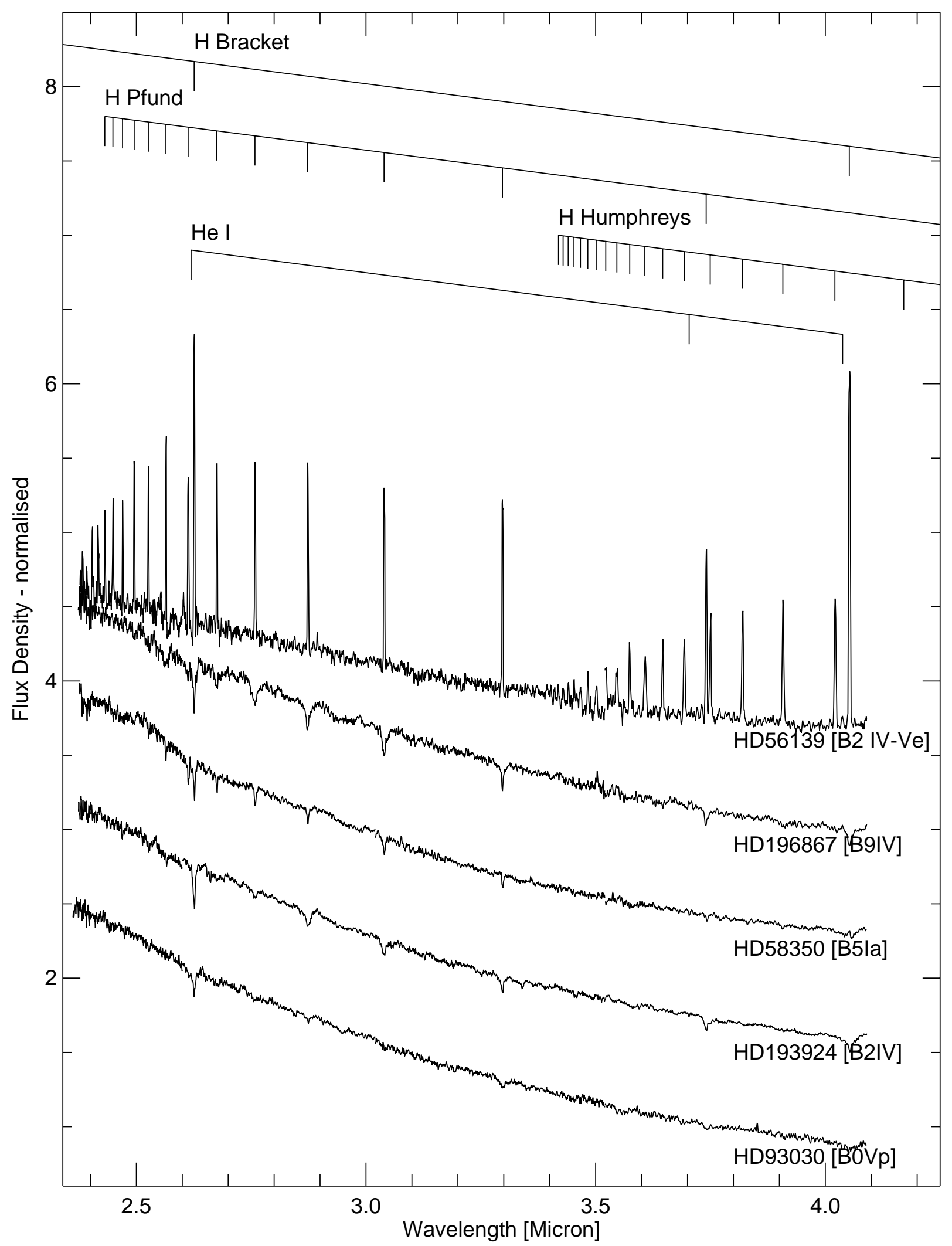

Fig. 10. Spectral features in the spectra of B and Be stars. The flux scale is in Jy, normalised to 1 at $3.8 \mu \mathrm{m}$ and offset for the different spectra.

$(2.36-3.8 \mu \mathrm{m})$ and the onset of the $\mathrm{SiO}$ first overtone (beyond $4.00 \mu \mathrm{m})$ The strength of the bands as we see them does not only depend on the fundamental stellar parameters. The emission from the circumstellar dust can fill in and weaken the photospheric absorption bands considerably. In M giants the circumstellar gas can contribute to an additional absorption or emission (Tsuji et al. 1997). Figure 15 depicts a number of cool giants ranging from $\mathrm{K} 0$ to $\mathrm{M} 8$ in spectral type. 

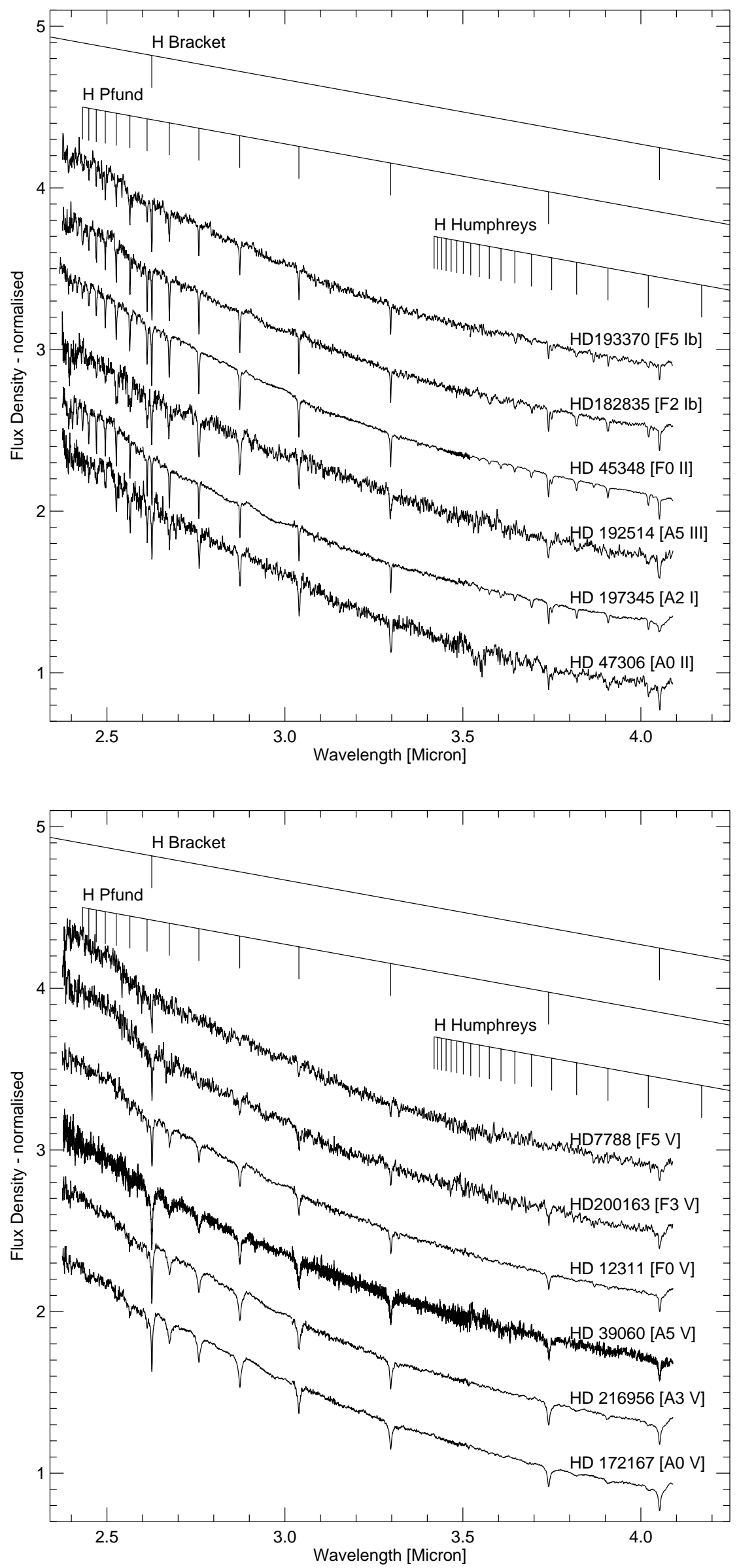

Fig. 11. Spectral features in the spectra of $A$ and $F$ giants and supergiants. The flux scale is in Jy, normalised to 1 at $3.8 \mu \mathrm{m}$ and offset for the different spectra.

Fig. 12. Spectral features in the spectra of $A$ and $\mathrm{F}$ dwarfs. The flux scale is in Jy, normalised to 1 at $3.8 \mu \mathrm{m}$ and offset for the different spectra. 

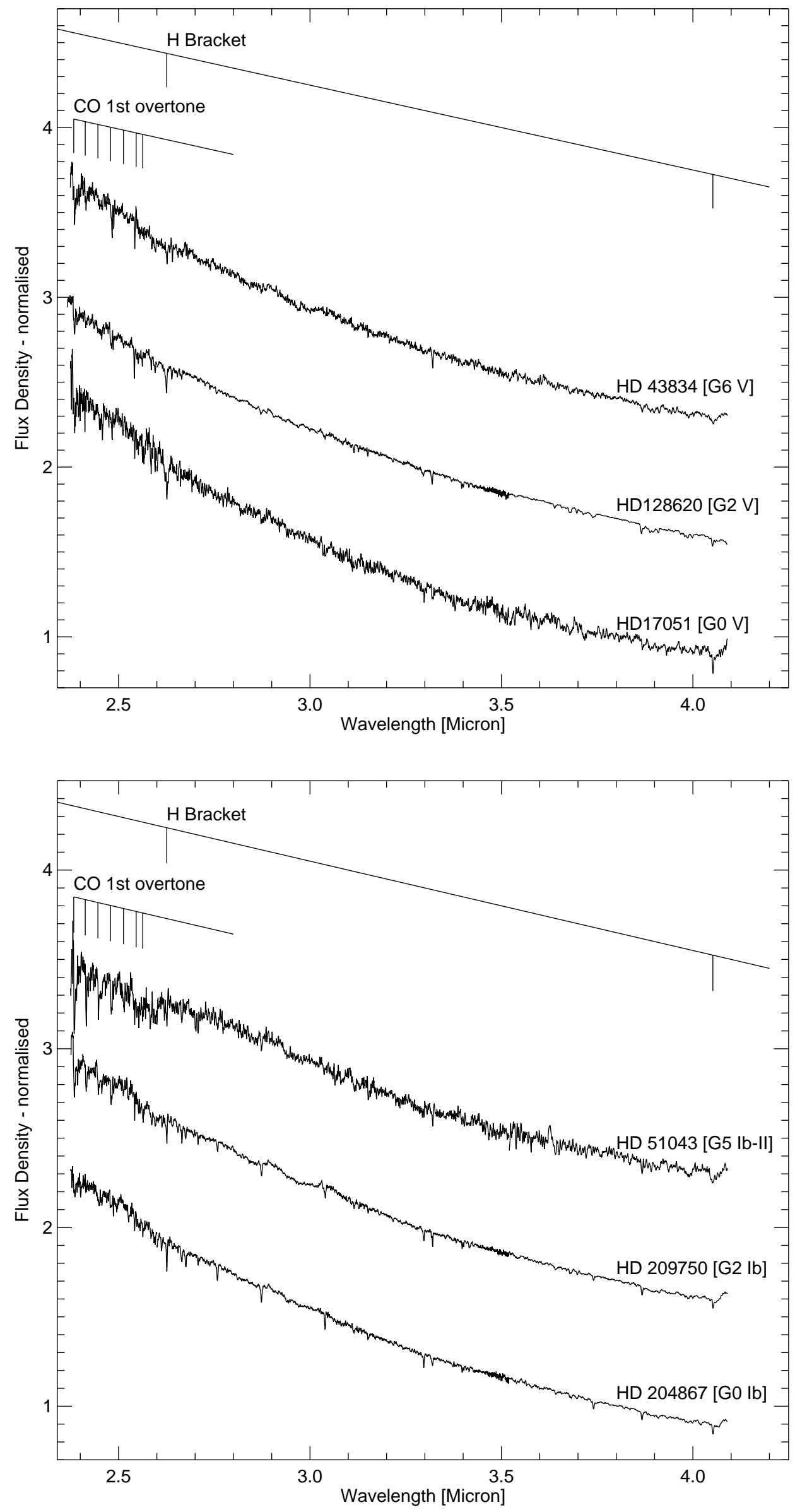

Fig. 13. Spectral features in the spectra of $\mathrm{G}$ dwarfs. Only the band-heads of the $\mathrm{CO}$ molecular band are indicated, although many fainter lines over the band are blended and contribute to a substantial depression of the continuum opacity. The flux scale is in Jy, normalised to 1 at $3.8 \mu \mathrm{m}$ and offset for the different spectra.

Fig. 14. Spectral features in the spectra of $\mathrm{G}$ supergiants. The flux scale is in Jy, normalised to 1 at $3.8 \mu \mathrm{m}$ and offset for the different spectra. 


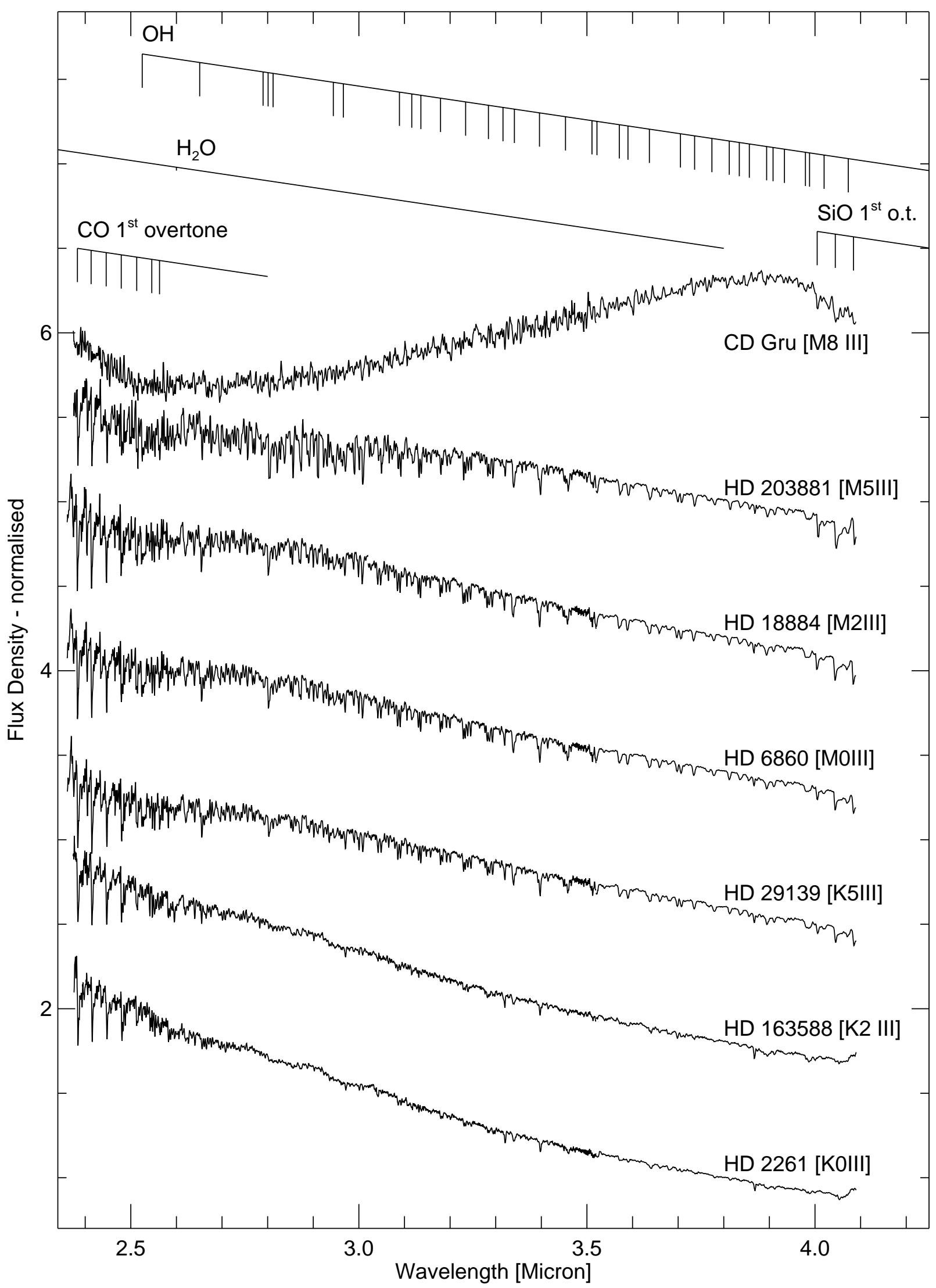

Fig. 15. Spectral features in the spectra of $\mathrm{K}$ and $\mathrm{M}$ stars. The flux scale is in Jy, normalised to 1 at $3.8 \mu \mathrm{m}$ and offset for the different spectra. Only the strongest lines of the $\mathrm{OH}, \mathrm{CO}$ and $\mathrm{SiO}$ molecular bands are indicated. A multitude of fainter lines in these bands and in the $\mathrm{H} 2 \mathrm{O}$ band are not resolved and contribute to a substantial depression of the continuum. 
Table 5. Overview of the spectra in the atlas of stars with spectral types earlier than A0.

\begin{tabular}{|c|c|c|c|c|c|c|}
\hline Spectral type & $\mathrm{V}$ & IV & III & II & I, Ia, Ib \& Iab & other \\
\hline $\mathrm{O} 4$ & & & & & 1 & \\
\hline O6 & 1 & & & & & \\
\hline O6.5 & 1 & & & & & \\
\hline O7.5 & & & & & 1 & \\
\hline O9 & 2 & & & & 1 & \\
\hline O9.5 & 1 & & & & 3 & \\
\hline O9.7 & & & & & 1 & \\
\hline B0 & 3 & 1 & & & & \\
\hline B 0.5 & 1 & & 1 & & 1 & \\
\hline B1 & 2 & & 1 & & 2 & \\
\hline B1.5 & & 1 & 1 & & 1 & \\
\hline B2 & 3 & 3 & 1 & & & 1 \\
\hline B2.5 & 3 & & & & 1 & \\
\hline B3 & 1 & 1 & 2 & & & \\
\hline B5 & 1 & 1 & 1 & 1 & 2 & \\
\hline B6 & 2 & & 1 & & & \\
\hline B7 & & 2 & & & 1 & \\
\hline B8 & 2 & & 1 & & 2 & \\
\hline B9 & 2 & 1 & 1 & & 1 & 1 \\
\hline B & & & & & & 7 \\
\hline WC & & & & & & 6 \\
\hline WN & & & & & & 8 \\
\hline PN & & & & & & 3 \\
\hline novae & & & & & & 2 \\
\hline supernovae & & & & & & 1 \\
\hline
\end{tabular}

\subsection{Carbon stars}

Secchi (1868) was the first to identify the class of carbon stars among red giants based on the presence of $\mathrm{C}_{2}$ lines in the optical spectrum. Wallerstein \& Knapp (1998) reviews what we have learned about carbon stars since then. The optical spectra also show lines of $\mathrm{CH}, \mathrm{CN}$, heavy elements like $\mathrm{Tc},{ }^{12} \mathrm{C}$ and ${ }^{13} \mathrm{C}$. Various theories exist on the mixing mechanisms that bring those elements to the surface. Many carbon giants on the Asymptotic Giant Branch (AGB) are variable and show a high mass loss. The circumstellar dust shell obscures their photospheres at visible wavelengths and causes an excess at midinfrared and far-infrared wavelengths.

At near-infrared wavelengths the circumstellar dust is optically thin and we see a broad absorption feature centred around $3.05 \mu \mathrm{m}$. This band is due to the stretching modes of $\mathrm{C}_{2} \mathrm{H}_{2}$ and HCN. The strength and shape of the bands associated with several vibrational transitions of $\mathrm{HCN}(2.5 \mu \mathrm{m}, 3.6 \mu \mathrm{m}, 3.85 \mu \mathrm{m})$ and $\mathrm{C}_{2} \mathrm{H}_{2}(2.6 \mu \mathrm{m}, 3.8 \mu \mathrm{m})$ varies from source to source. We also recognise the $\mathrm{CO}$ first overtone band around $2.5 \mu \mathrm{m}$, the $\mathrm{CH}$ fundamental band (3-4 $\mu \mathrm{m})$ and the CS first overtone band around $4 \mu \mathrm{m}$ (Aoki et al. 1998). Figure 16 shows three carbonrich objects with the important molecular bands indicated.

\section{Electronic availability of the data}

The reduced data have been added to the data archive at the ISO Data Centre ${ }^{2}$. The complete atlas is available in elec-

\footnotetext{
${ }^{2}$ http://www.iso.vilspa.esa.es
}

Table 6. Overview of the atlas spectra of stars with spectral types later than A0.

\begin{tabular}{|c|c|c|c|c|c|c|}
\hline Spectral type & $\mathrm{V}$ & IV & III & II & I, Ia, Ib \& Iab & other \\
\hline $\mathrm{A} 0$ & 2 & 1 & 1 & 1 & & \\
\hline A1 & & & & & 1 & \\
\hline A2 & & & 1 & & 3 & \\
\hline A3 & 1 & & & & 2 & \\
\hline A5 & 2 & & 1 & & & 1 \\
\hline A7 & 3 & & & & & \\
\hline A8 & 1 & & 1 & & & 1 \\
\hline A9 & 2 & 1 & & & & \\
\hline F0 & 1 & 1 & 1 & 1 & & \\
\hline F2 & 2 & 1 & & & 3 & 1 \\
\hline F3 & 1 & 3 & 2 & & 1 & \\
\hline F4 & 1 & & & & & \\
\hline F5 & 1 & & 1 & 1 & 3 & \\
\hline F6 & & 1 & & 1 & 2 & \\
\hline F7 & 4 & & & 1 & 2 & \\
\hline F8 & 1 & 1 & & & & 4 \\
\hline G0 & 2 & & 1 & & 2 & \\
\hline G1 & & 1 & & & & \\
\hline G2 & 3 & 1 & 1 & & 1 & \\
\hline G3 & 1 & 1 & & & 1 & \\
\hline G4 & 1 & & & & 1 & \\
\hline G5 & 1 & & & & 2 & \\
\hline G6 & 1 & 1 & & & 1 & \\
\hline G7 & & 1 & & & 1 & \\
\hline G8 & 1 & 1 & 4 & & 1 & \\
\hline G9 & & & 2 & & & \\
\hline K0 & 1 & 1 & 3 & 1 & 1 & \\
\hline $\mathrm{K} 1$ & 1 & & 1 & & 1 & \\
\hline K1.5 & & & 1 & & & \\
\hline $\mathrm{K} 2$ & & & 1 & & 1 & \\
\hline K3 & & & 2 & 1 & 1 & \\
\hline K4 & 2 & & 2 & & 1 & \\
\hline K5 & 1 & & 4 & & 1 & \\
\hline K7 & 1 & & 1 & & 1 & \\
\hline M0 & & & 1 & & & \\
\hline M1 & 1 & & 1 & & 2 & \\
\hline M2 & & & 1 & & 2 & \\
\hline M2.5 & & 1 & & & & \\
\hline M3 & & & 2 & 1 & 2 & \\
\hline M4 & & & 1 & 1 & & 1 \\
\hline M5 & & & 5 & 2 & & 2 \\
\hline M6 & & & 5 & & & \\
\hline M7 & & & 4 & & & 1 \\
\hline M7.5 & & & & & & 1 \\
\hline M8 & & & 2 & & & 2 \\
\hline M9 & & & & & & 1 \\
\hline $\mathrm{S}$ & & & & & & 2 \\
\hline $\mathrm{C}$ & & & & 8 & & 8 \\
\hline
\end{tabular}

tronic form as a PDF file at the EDP Sciences A\&A website at http://www.edpsciences.fr. In the atlas we have ordered the spectra of our sample according to spectral type as found in the literature. For each star we have listed some relevant literature values at the bottom of the page. We list the source's HD number if available, together with other names of the source. The spectral type is listed if available. For some stellar sources like novae or planetary nebulae we have not 


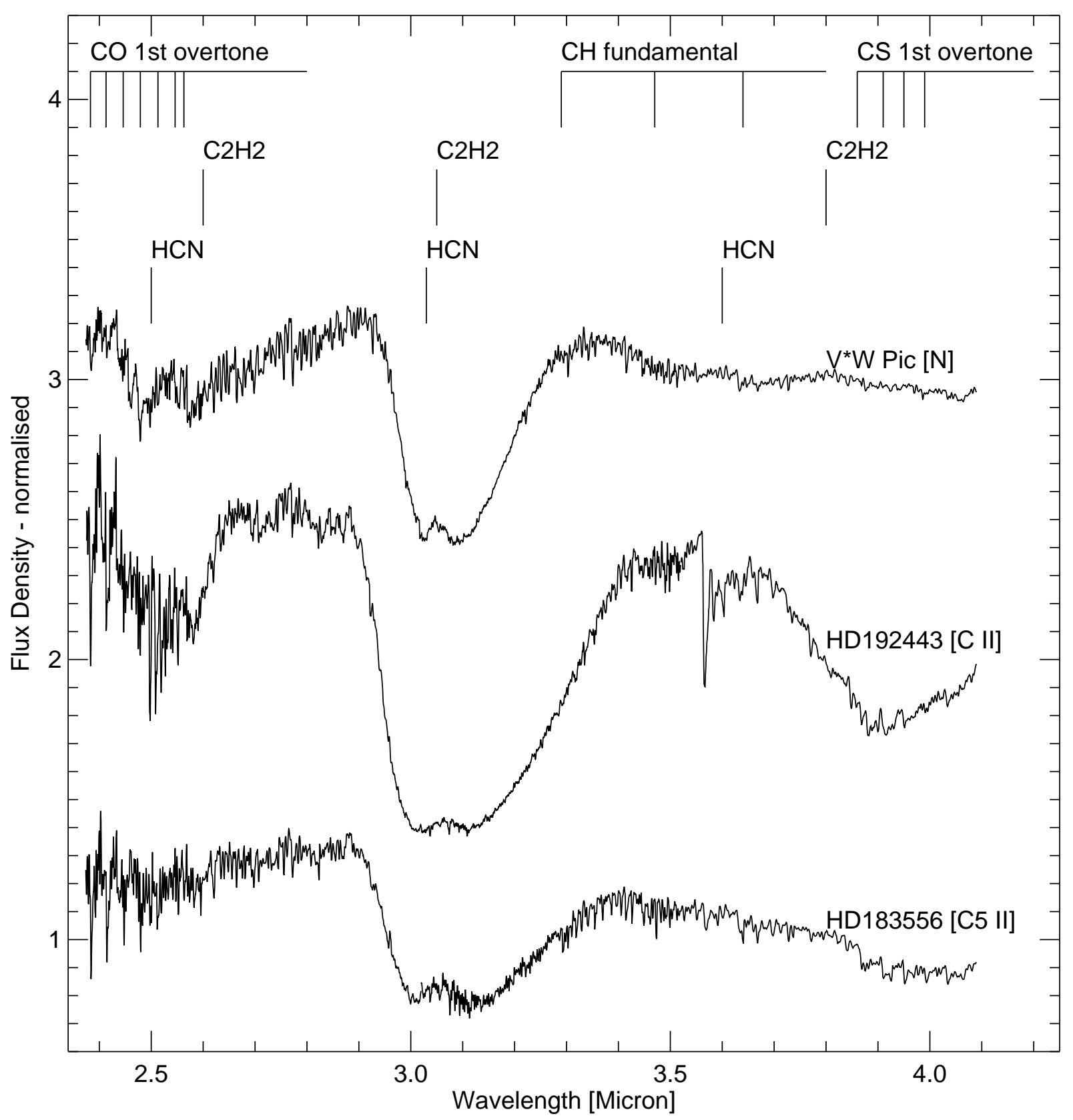

Fig. 16. Spectral features in the spectra of carbon stars: the flux scale is in Jy, normalised to 1 at $3.8 \mu \mathrm{m}$ and offset for the different spectra. Only the strongest lines of the $\mathrm{CO}, \mathrm{CS}$ and $\mathrm{CH}$ molecular bands have been indicated.

specified the MK-classification here, but rather a description of the nature of the source.

For every source the $V$ magnitude and $B-V$ colour is given. If available, we have listed the Hipparcos magnitudes and colours here. If not, other values from the literature are taken. The IRAS number of the source in the Point Source Catalog is listed together with the in-band fluxes at 12, 25, 60 and $100 \mu \mathrm{m}$. Unfortunately, the 1986 edition of the PSC does not indicate if the flux densities are flux densities or upper limits for the faintest sources. Care should be taken when interpreting low flux values. We also list the ISO observation identification (TDT) which identifies the plotted observation uniquely in the ISO Data Archive.
Furthermore, we indicate relevant positional properties: Right Ascension [RA], Declination [Dec], Proper Motion in Right Ascension [pm(RA)], Proper Motion in declination $[\mathrm{PM}(\mathrm{dec})]$ and parallax. From these values and the spacecraft attitude control system data we have calculated the offset between the source position and the center of the SWS slit. The values are calculated in the coordinate frame of the spacecraft $y$-and $z$-axis. These correspond to the cross-dispersion and the dispersion direction of the SWS instrument.

\section{Conclusion}

The ISO-SWS observations obtained after the liquid helium depletion have resulted in a unique atlas of $2.36-4.05 \mu \mathrm{m}$ spectra 
of stars. The drifts in the instrument characteristics are smooth and can be calibrated accurately with the calibration observations performed in the course of the programme. The spectra obtained result in a spectral atlas covering the entire MK classification scheme. The dataset is the basis of two forthcoming papers by Lenorzer et al. (2002) and Vandenbussche et al. (in prep.) in which the near-infrared signature of stars is linked quantitatively to the fundamental properties of the stars.

Acknowledgements. LBFMW acknowledges financial support through an NWO Pionier grant. This research has made use of the SIMBAD and Vizier databases, operated at CDS, Strasbourg, France. The authors wish to thank P. Morris for his work on the definition of the post-helium programme; D. Lutz and R. Shipman are acknowledged for their suggestions that led to several improvements in the manuscript.

\section{References}

Aoki, W., Tsuji, T., \& Ohnaka, K. 1998, A\&A, 340, 222

Cohen, M., Walker, R. G., Carter, B., et al. 1999, AJ, 117, 1864

de Graauw, T., Haser, L. N., Beintema, D. A., et al. 1996, A\&A, 315, L49

Decin, L. 2000, Ph.D. Thesis, Katholieke Universiteit Leuven

Decin, L., Vandenbussche, B., Waelkens, C., et al. 2002a, A\&A, submitted

Decin, L., Vandenbussche, B., Waelkens, C., et al. 2002b, A\&A, submitted

Decin, L., Vandenbussche, B., Waelkens, C., et al. 2002c, A\&A, submitted

Decin, L., Waelkens, C., Eriksson, K., et al. 2000, A\&A, 364, 137

Förster Schreiber, N. M. 2000, AJ, 120, 2089

Hoffleit, D., \& Warren, J. W. H. 1991, The Bright Star Catalogue 5 th revised ed. (Astronomical Data Center, NSSDC/ADC), ISBN: 9290923997
Kessler, M. F., Steinz, J. A., Anderegg, M. E., et al. 1996, A\&A, 315, L27

Lançon, A., \& Wood, P. R. 2000, A\&AS, 146, 217

Leech, K., Kester, D., Shipman, R., et al. 2002, The ISO Handbook, Vol. V: SWS - The Short Wavelength Spectrometer, v2.0, ISO Data Centre, Astrophysics division, Space Science Department of ESA, VILSPA, PO Box 50727, E-28080 Madrid

Leech, K., \& Pollock, A. 2001, The ISO Handbook, vol II, ISO Data Centre, Astrophysics division, Space Science Department of ESA, VILSPA, PO Box 50727, E-28080 Madrid

Lenorzer, A., Vandenbussche, B., Morris, P., et al. 2002, A\&A, 384, 473

Meyer, M. R., Edwards, S., Hinkle, K. H., \& Strom, S. E. 1998, ApJ, 508, 397

Morgan, W. W., Keenan, P. C., \& Kellman, E. 1943, in Astrophysics monographs University of Chicago Press

Morris, P. W., Bouwman, J., Crowther, P. A., et al. 1999, in ESA SP427: The Universe as Seen by ISO, 427, 247

Olnon, F. M., Raimond, E., Neugebauer, G., et al. 1986, A\&AS, 65, 607

Price, S. D., Sloan, G. C., \& Kraemer, K. E. 2002, ApJ, 565, L55

Ryde, N., \& Eriksson, K. 2002, A\&A, 386, 874

Secchi, A. 1868, MNRAS, 28, 196

Tsuji, T. 2001, A\&A, 376, L1

Tsuji, T., Ohnaka, K., Aoki, W., \& Yamamura, I. 1997, A\&A, 320, L1

Valentijn, E. A., Feuchtgruber, H., Kester, D. J. M., et al. 1996, A\&A, 315 , L60

van der Hucht, K. A., Conti, P. S., Lundstrom, I., \& Stenholm, B. 1997, VizieR Online Data Catalog, 3085

van der Hucht, K. A., Morris, P. W., Williams, P. M., et al. 1996, A\&A, 315, L193

Wallace, L., \& Hinkle, K. 1996, ApJS, 107, 312

Wallace, L., Meyer, M. R., Hinkle, K., \& Edwards, S. 2000, ApJ, 535, 325

Wallerstein, G., \& Knapp, G. R. 1998, ARA\&A, 36, 369

Willis, A. J., Dessart, L., Crowther, P. A., et al. 1997, MNRAS, 290, 371 